\title{
An immunoinformatics approach for the design of a multi- epitope subunit vaccine for urogenital schistosomiasis
}

\author{
Olugbenga S Onile ${ }^{\text {Corresp., }}{ }^{1}$, Adeyinka I Fadahunsi ${ }^{1}$, Ameerah A Adekunle ${ }^{1}$, Bolaji Fatai Oyeyemi $^{2}$, Chiaka I \\ Anumudu $^{3}$ \\ 1 Biotechnology Programme, Department of Biological Sciences, Elizade University, Ilara-Mokin, Ondo State, Nigeria \\ 2 Molecular Biology Group, Department Science Technology, The Federal Polytechnic, Ado-Ekiti, Ado-Ekiti, Ekiti State, Nigeria \\ 3 Cellular Parasitology Programme, Department of Zoology, University of Ibadan, Ibadan, Oyo State, Nigeria \\ Corresponding Author: Olugbenga S Onile \\ Email address: olugbenga.onile@elizadeuniversity.edu.ng
}

Discovery of $\mathrm{T}$ and $\mathrm{B}$ memory cells capable of eliciting long-term immunity against schistosomiasisis is important for people in endemic areas. Changes in schistosomes environment due to developmental cycle, induces up-regulation of Heat Shock Proteins (HSPs) which assist the parasite in coping with the hostile conditions associated with its life cycle. This study therefore focused on exploring the role of HSPs in urogenital schistosomiasis to develop new multi-epitope subunit vaccine against the disease using immunoinformatic approaches. The designed subunit vaccine was subjected to in silico antigenicity, immunogenicity, allergenicity and physicochemical parameters analysis. A 3D structure of the vaccine construct was predicted, followed by disulphide engineering for stability, codon adaptation and in silico cloning for proper expression and molecular protein-protein docking of vaccine construct in the vector against TLR4 receptor respectively. Consequently, a 493 amino acid multi-epitope vaccine construct of antigenicity probability of 0.91 was designed. This was predicted to be stable, nonallergenic in nature and safe for human use. 
1 2

3 Onile Olugbenga S. ${ }^{*}$, Fadahunsi Adeyinka I. ${ }^{1}$, Adekunle Ameerah A. ${ }^{1}$, Oyeyemi Bolaji $\mathrm{F}^{2}$. 4 Anumudu Chiaka I. ${ }^{3}$

5 'Biotechnology Programme, Department of Biological Sciences, Elizade University, Ilara6 Mokin, Nigeria; ${ }^{2}$ Molecular Biology Group, Department of Science Technology, The Federal 7 Polytechnic, Ado-Ekiti, Nigeria; ${ }^{3}$ Cellular Parasitology Programme, Department of Zoology, 8 University of Ibadan, Ibadan, Nigeria.

9 *Corresponding Author's Address:

10 Olugbenga Samson Onile

11 Email: olugbenga.onile@elizadeuniversity.edu.ng; onileg@yahoo.com

12 Phone Number: +2348133109389 


\section{Abstract}

32 Discovery of $\mathrm{T}$ and $\mathrm{B}$ memory cells capable of eliciting long-term immunity against schistosomiasisis is important for people in endemic areas. Changes in schistosomes environment due to developmental cycle, induces up-regulation of Heat Shock Proteins (HSPs) which assist the parasite in coping with the hostile conditions associated with its life cycle. This study therefore focused on exploring the role of HSPs in urogenital schistosomiasis to develop new multi-epitope subunit vaccine against the disease using immunoinformatic approaches. The designed subunit vaccine was subjected to in silico antigenicity, immunogenicity, allergenicity and physicochemical parameters analysis. A 3D structure of the vaccine construct was predicted, followed by disulphide engineering for stability, codon adaptation and in silico cloning for proper expression and molecular protein-protein docking of vaccine construct in the vector against TLR4 receptor respectively. Consequently, a 493 amino acid multi-epitope vaccine construct of antigenicity probability of 0.91 was designed. This was predicted to be stable, non-

44 allergenic in nature and safe for human use. 
Introduction

59

60

61

62

63

64

65

66

67

68

69

70

71

72

73

74

75

76

77

78

79

80

Urinary schistosomiasis has been identified as the main cause of urogenital disease, and the main cause of bladder cancer in developing countries (Onile et al. 2016; Rollinson et al. 2013). In 76 countries, more than 200 million people are estimated to be affected by urogenital schistosomiasis with more than 100 million being urogenital schistosomiasis. In Sub-Saharan Africa, mortality rate of the disease is estimated at 250,000 persons per year (Masamba et al. 2016). In the year 2008, estimated cases of 29 million infected persons were reported in Nigeria which amount to the largest number of infections in Africa (Adenowo et al. 2015) and may have increased (Abdulkadir et al. 2017). Bergquist (2013) reported the spread of the disease to regions that were previously non-endemic. These include reported cases of urinary schistosomiasis in adolescent refugee from Gambia (Poddighe et al. 2016) and 214 male migrant patients Milesi et al. (2019) all in Italy. Uysal et al. (2014) also reported a rare case of a 22 year old Nigerian with urinary schistosomiasis in Turkey. Causes of several form of bladder pathologies and tumours in Africa including Nigeria has been linked to urogenital schistosomiasis (Adebayo et al. 2017; Barsoum 2013; Onile et al. 2016). Praziquantel has been the most used drug for treating schistosomiasis since the early 2000s (Chevalier et al. 2016; Doenhoff et al. 2008) but the drug has been found to be less effective against the worm in all stages of its life cycle and would not eliminate immature worms within 3-4weeks of infection (Alsaqabi \& Lotfy 2014; Aragon et al. 2009; Cioli et al. 2014; Doenhoff et al. 2008). Also several cases of drug resistance to praziquantel due to pressure from continuous mass drug administration have now been reported (Couto et al. 2011; Fallon et al. 1996; Melman et al. 2009).

79 By transiting between intra-mammalian, aquatic and snail stages to develop into full maturity, schistosomes face a number of hostile environments throughout their lifecycle (Mbah et al. 2013) 
81 and heat shock responses have been associated with cellular stress during the movement of parasite from a cooler, low saline and freshwater environment to a warmer, saline environment of a human host (Devaney 2006; Mbah et al. 2013). Heat shock proteins (HSP) are an important group of molecules involved in a range of biological and developmental processes in schistosomes and other parasites (Braschi et al. 2006). Hsp 90 and other HSPs are chaperone proteins that assist proper folding of other proteins. They stabilize proteins against heat stress, and alos aid protein degradation. Other functions include stabilizing plethora of proteins required for tumour growth; therefore HSP inhibitors are being investigated as vaccines and anti-cancer drugs (Das et al. 2019). Identification of HSPs in cercarial gland secretions and the highest abundance of the protein transcripts in newly transformed schistosomula have been reported (Ishida \& Jolly 2016). The role of heat shock response in snail susceptibility to Schistosoma infection in the intermediate molluscan host has also been emphasized (Ishida \& Jolly 2016). (Onile et al. 2017), had reported several HSPs as possible biomarkers for the diagnosis of urinary schistosomiasis, they are also known to play critical roles in innate immunity and adaptive immunity. HSPs can activate specific toll like receptors (TLRs), provide polypeptides for specific triggering of the acquired immune response and, play a major role in cross-presentation of extracellular antigens. This would result in the induction of CD8+ cytotoxic T-lymphocyte responses (McNulty et al. 2013). Antigen presenting cells (APCs) are known for recognizing a wide range of molecular patterns expressed by pathogens called pathogen-associated molecular patterns (PAMPs) during innate immune responses (El-Din 2016; Venugopal et al. 2009). Toll-like receptors (TLRs) and NOD-like receptors (NLRs) through signaling pathways that induce upregulation of IFNs and other inflammatory cytokines are used by APCs to recognize these PAMPs (Venugopal et al. 2009). (Zhang et al. 2011) had reported mice with deficient TLR after infection with $S$. japomicum showed increasing level of parasite egg load.

HSPs were chosen in this study as target for the development of a new multi-epitope subunit vaccine against urinary schistosomiasis for their role in Schistosoma biology. .

\section{Methodology}


109

110

111

112

113

114

115

116

117

118

119

120

121

122

123

124

125

126

127

128

129

130

131

132

133

134

135

136

137

138

\section{Retrieval of Schistosoma haematobium heat shock protein (HSP) sequence for vaccine} construction

The amino acid sequences of Schistosoma haematobium HSPs were retrieved from the NCBI protein database (www.ncbi.nlm.nih.gov) and subjected to multiepitope vaccine designing. To test for the proteins ability to induce immune response within a host body, all the retrieved protein sequences were subjected to an antigenicity test using the ANIGENpro database (scratch.proteomics.ics.uci.edu). Antigenic probability of $\geq 0.8$ was used to determine which proteins to be chosen for the next step of the multi epitope vaccine construct (Pandey et al. 2018).

\section{Prediction of Cytotoxic T-Lymphocytes (CTL) and helper T-Lymphocytes (HTL) epitope} and immunogenicity

To get an immunogenic CTL epitope with potential of inducing cell mediated immunity and form memory cells, all the three highly antigenic HSP sequences in FASTA format were fed into the NetCTL 1.2 server (http://www.cbs.dtu.dk/services/NetCTL/) to predict the CTL epitope at threshold score of 0.75 (using the default setting). The server predicts CTL epitopes from inputted protein sequences based on the training dataset (prediction of MHC-I binding peptides, proteasomal C-terminal cleavage, and TAP (Transporter Associated with Antigen Processing) transport efficiency) and only epitopes with combined score greater than 0.75 were selected as CTL epitopes (Khatoon et al. 2017; Pandey et al. 2018). The selected CTL epitopes (Scores $>0.75$ ) were further analysed in Immune Epitope Design Database (www.iedb.org) for MHC class I immunogenicity prediction. The immunogenicity score determines the probability of eliciting an immune response (higher score means greater probability of immune response and vice versa) (Pandey et al. 2018).

The HTL epitopes of $15 \mathrm{mer}$ length for mouse allele (H2-IAb, H2-IAd and H2-IEd) were predicted for the HSPs of $S$. haematobium using the Immune Epitope Design Database. The output epitopes were ranked based on lower percentile rank scores i.e the lower the ranked score the higher the binding affinity for HTL receptors (Khatoon et al. 2017). Only epitopes with higher binding affinity $(\leq 1.5)$ with MHC-II were selected for the final multi-epitope vaccine construct. In order to determine the ability of the predicted epitopes in activating Th1 type immune response followed by IFN- $\gamma$ production, the top 17 HTL epitopes were subjected in 
139 FASTA format to the IFN epitope server by using the Motif and SVM hybrid as approach

140 selection and IFN- $\gamma$ versus other cytokine as model of prediction.

\section{Construction of multi-epitope subunit vaccine}

142 To design a viable vaccine construct capable of inducing innate and adaptive immune response,

143 the high scoring CTL and HTL epitopes from the present immunoinformatics predictions were 144 used. These epitopes were joined together using AAY and GPGPG linkers which were added at 145 the intra-epitope position thereby linking the CTL and HTL epitopes respectively (Khatoon et al. 146 2017). Also, a TLR-4 agonist (RS-09; Sequence: APPHALS) was used adjuvant (Pandey et al. 147 2018; Shanmugam et al. 2012) and linked with the multi-epitopes (CTL and HTL) using 148 EAAAK linker (Lee et al. 2014).

149 B Cell epitopes prediction for Schistosoma haematobium proteins

150 The BCPREDS server (ailab.ist.psu.edu/bcpred) was used to predict the linear B-cell epitopes for 151 the final vaccine construct. The 493 amino acid sequence of the final vaccine construct was 152 inputted into the BCPREDS server in plain format followed by the selection of a fixed length 153 epitope prediction method and length of the epitope. The default method of BCPREDS was 154 selected as the prediction method for the amino acid epitopes (El-Manzalawy et al. 2008; Pandey 155 et al. 2018). The specificity threshold was set as default at 75\% and conformational epitopes 156 were predicted using the ElliPro server for the tertiary protein structure of the vaccine construct 157 (Pandey et al. 2018).

158

159

160

161

162 163

164 165

166 167

\section{Prediction of antigenicity, allergenicity and physiochemical parameter of vaccine construct}

The potential of the vaccine construct in eliciting immunological response through binding to the $\mathrm{B}$ and $\mathrm{T}$ cell receptor was determined by first determining the antigenicity of the vaccine construct. The ANTIGENpro server (scratch.proteomics.ics.uci.edu); a sequence-based alignment free and pathogen independent predictor was used to generate the antigenicity index (Khatoon et al. 2017).

The non-allergenic potential of the multi-epitope vaccine construct was determined using the AllerTOP v.2.0 server (www.ddg-pharmfac.net/AllerTOP). Several physiochemical parameters of the vaccine construct which include amino acid composition, the theoretical pI, instability index, in-vitro and in-vivo half-life, aliphatic index, molecular weight, and grand average of 
168 hydropathicity (GRAVY) parameters were all assessed using the ProtParam server 169 (https://web.expasy.org/protparam/) (Onile 2014).

170 Prediction, refinement and validation of the tertiary structure of vaccine construct

171 The 3D structure of the vaccine construct was predicted using RaptorX structure prediction 172 server (raptorx.uchicago.edu/). This server predicts secondary and tertiary structures as well as 173 contact map, solvent accessibility, disordered regions and binding sites of protein sequences. 174 RaptorX server also assign some confidence scores P-value, GDT (global distance test) and 175 uGDT (un-normalized GDT), and modeling error at each residue to indicate the quality of a 176 predicted 3D mode. In order to improve and refine the predicted 3D model of vaccine construct, 177 the output model of RaptorX server was further modelled using GalaxyRefine server 178 (http://galaxy.seoklab.org/) (Heo et al. 2013). This server uses CASP10 based refinement 179 approach to reconstruct and repack the protein side chain, followed by dynamics simulation to 180 relax the structure. ProSA-wed (https://prosa.services.came.sbg.ac.at/prosa.php) was employed 181 in the tertiary structure validation; also the refinement output was validated using Ramachandran 182 plot analysis (Mordred.bioc.cam.au.ck/ rapper/rampage.php).

\section{Disulfide engineering for vaccine stability}

184 The Disulfide by Design v2.12 server was used for the in silico disulfide engineering of the refined model 3D structure of the vaccine construct. The refined model was first run to determine residue pairs useful for the purpose of disulphide engineering. Only residue pairs with energy

$187(\mathrm{kcal} / \mathrm{mol})$ less than 2.2 and $\mathrm{Chi}^{3}\left(\chi^{3}\right)$ between -87 and +97 degree were selected for mutation 188 (Rana \& Akhter 2016). A total of 5 residue pairs were eventually selected for mutation by using 189 the create mutate icon of the Disulfide by Design server.

190 Codon adaptation and in silico cloning

191 To determine and optimise the expression rate of the vaccine construct in a proper expression 192 vector, the primary sequence of the vaccine construct was inputted into the Java Codon 193 Adaptation Tool (JCAT) for codon optimization which was performed in the host E. coli strain $194 \mathrm{~K} 12$. Due to difference in codon usage of E. coli and native host S. haematobium from where the 195 vaccine construct sequences originated, three different options in the JCAT server were selected 196 which include: avoid rho-independent transcription, prokaryotes ribosome binding site and 197 restriction enzymes cleavage sites. To determine the high-level of protein expression, the output 
198 of the JCAT which include codon adaptation Index and percentage GC content were used. E. coli

199 pET-28(+) was used as vector to clone the adapted nucleotide sequence (provided by JCAT 200 server) of the final vaccine construct using the restriction cloning module of SnapGene tool (Ali 201 et al. 2017).

\section{Molecular docking of vaccine construct with TLR4}

203 The ClusPro 2.0 server was used for the molecular protein-protein docking to check for the 204 binding affinity between the vaccine construct and TLR-4 receptor (Kozakov et al. 2017; Pandey 205 et al. 2018). The refined sub unit multi-epitope vaccine protein and TLR-4 PDB file (4G8A) 206 obtained from the RCSB-Protein Data Bank were used as the ligand and receptor respectively.

\section{Results}

209

210

211

212

213

214

215

216

217

218

219

220

221

222

223

224

225

\section{Retrieved $S$. haematobium HSP sequences for vaccine construct}

To design the multi-epitope subunit vaccine for urinary schistosomiasis consisted of seven (7) HSP sequences [HSP90 (KGB37337.1), HSP11 (XP_012799478.1), HSP97 (KGB34481.1), HSP75 (KGB38609.1), HSP83 (KGB37622.1), HSP10 (KGB41273.1), HSP60 (KGB40455.1)] were retrieved from the NCBI database (https://www.ncbi.nlm.nih.gov) in FASTA format. Only three (HSP11, HSP97, HSP90) proteins had an antigenic probability of $\geq 0.8$ after retrieved protein sequences were analyzed using ANTIGENpro for antigenicity prediction (Table 1), consewuently they were selected for the vaccine construct.

\section{Predicted Cytotoxic T-Lymphocytes (CTL), Helper T-Lymphocytes (HTL) epitope and} immunogenicity

By using the NetCTL 1.2 server for prediction of the CTL receptor specific immunogenic epitopes, a total of 23 CTL epitopes (9mer length) were obtained for the inputted three HSP protein sequences and only 12 epitopes with high immunogenicity scores as obtained from IEDB server were chosen as the final CTL epitopes to undergo vaccine designing (Table 2).

The prediction of the HTL epitope was carried out using the IEDB MHC-II epitope prediction module, all three protein sequences were subjected to the module. The mouse alleles used for prediction were H2-1Ad, H2-1Ed, and H2-1Ab. However, only 17 HTL epitopes with lowest 
226 percentile with $\leq 1.5$ with MHC-II were selected and subjected for the final multi-epitope vaccine

227 construct (Table 3a). Also, all the 17 HTL epitopes have the capacity of inducing IFN- $\gamma$, and this

228 was evident from the positive score obtained from the IFN epitope server output (Table 3b)

\section{Constructed Multi-epitope Subunit Vaccine}

230 The constructed multi-epitope subunit vaccine consisted of 12 CTL epitopes and 17 HTL 231 epitopes with high binding affinity fused together with the help of AAY, GPGPG and EAAAK 232 linkers. The adjuvant and the CTL epitopes were combined by the EAAAK linkers, the intra233 CTL and intra-HTL epitopes were joined by intra-CTL AAY and intra-HTL GPGPG linker 234 respectively to form the final vaccine constructs composed of 493 amino acid residues. For 235 maximum ability of the candidate vaccine to elicit adequate immune response, adjuvant and CTL 236 epitopes were joined together using an EAAAK linker (Figure 1a).

237

238

239

240

241

242

243

244

245

246

247

248

249

250

251

252

253

254

\section{Predicted B cell epitopes for Schistosoma haematobium proteins}

A total of 14 epitopes having 0.99 and above probability score with 20mers length were selected as the linear B cell binding epitopes for the final vaccine construct (Table 4). Discontinuous epitopes of 125 amino acids were predicted from the final 3D model of vaccine construct with the score of 0.734 (Figure 1b).

\section{Predicted antigenicity, allergenicity and physiochemical parameter of the vaccine construct}

The antigenicity test of the designed vaccine construct showed the antigenicity probability to be 0.905150, this represents the antigenic nature of the vaccine construct. An allergenicity test carried out for the vaccine construct also showed that the vaccine candidate is non-allergenic in nature.

The prediction of the physiochemical parameters of the vaccine construct was characterized by using the ProtParam server which evaluated for the following parameters; The molecular weight was $51409.68 \mathrm{kDa}$, theoretical pI 9.11, while the total number of negative and positive charge residues were 36 and 49 respectively. The estimated half-life was 4.4 hours in mammalian reticulocytes in-vitro, 20 and 10 hours in yeast and Escherichia coli respectively. The extinction coefficient was found to be $36790 \mathrm{M}^{-1} \mathrm{~cm}^{-1}$, at $280 \mathrm{~nm}$ measured in water assuming all pairs of cysteine residues are reduced, instability index was given to be 35.01. The value of the aliphatic index was 77.81 while the grand average of hydropathicity (GRAVY) was -0.138 . 
255 Predicted, refined and validated tertiary structure of vaccine construct

256 The 3D model and tertiary structure were predicted using RaptorX server (Figure 2). The best

257 template for the homology modeling was PDB ID: 5jalA with 493 (100\%) amino acid residues

258 modelled as single domain with $4 \%$ disorder. The secondary structure information includes helix

$25931 \%$, Beta sheet $17 \%$ and coiled structure 51\%. To refine the vaccine construct, GalaxyRefine

260 server was used. Out of all refined models, model 3 was selected as the best based on various

261 parameters including GDT-HA (0.9239), RSMD (0.484), MolProbity (2.69), Clash Score (32.7)

262 and Poor rotamers (1.9).

263 The refinement output was also validated using Ramachandran plot analysis, which revealed that

$26493.3 \%$ of the residue are located in most favoured regions, 5.3\% in allowed regions and only

$2651.4 \%$ in outlier region and the quality and potential errors in the crude 3D as verified by ProsSA-

266 web showed Z-scores of -6.01 for ProSA (Figure 3 and 4).

\section{Disulfide engineering for vaccine stability}

268 Disulfide engineering was done to stabilize the final model of the vaccine construct using 269 Disulfide by Design v2.12. The result showed a total 58 pairs of residues that are useful for the 270 purpose of disulphide engineering (Figure 5) but 10 mutations were created from the only 5 pairs 271 of residues (GLU56-CYS249, TYR60-HIS493, VAL116-GLN242, GLN243-GLU489) that were

272 found with energy value less than 2.2 and Chi3 value range between -87 and +97 degree

273 Codon adaptation and in silico cloning

274 The codon representing the subunit vaccine candidate was adapted to the codons of the E. coli $\mathrm{K}$

27512 strain using JCAT server and showed that the optimized codon sequence has a length of 1450

276 nucleotides with a codon adaptation index (CAI) of 0.95 and average GC content of $54.56 \%$. The

277 adapted codon sequence was later inserted into pET28(+) vector to form the restriction clone

278 (Figure 6). The adapted codon sequences used in insilico cloning are made up of the following $2791400 \quad$ nitrogenous bases

280 "GCTCCGCCGCACGCTCTGTCTGAAGCTGCTGCTAAACTGGACATGACCGAAGAATG

281 GCTGTACGCTGCTTACCAGACCGAAGAAATCGACGGTACCCTGGCTGCTTACTCTAT

282 CGCTGCTGGTGAACCGACCTACGCTGCTTACTCTCAGGCTCAGCTGATCGAAGAATA

283 CGCTGCTTACAACTCTAAAAACGCTGTTGAAGAATACGCTGCTTACTTCACCGTTAT

284 CGAACAGTGCCTGTACGCTGCTTACGGTTCTGACGGTTGCTTCGTTTCTTTCGCTGCT 
285

286

287

288

289

290

291

292

293

294

295

296

297

298

299

300

301

302

303

304

305

306

307

308

309

310

311

312

TACTTCTCTGGTAACATCACCGGTATCTTCGCTGCTTACAAACTGGACGGTGAAGCT AACGTTTACGCTGCTTACGGTTCTAAAATCGAAAACGACCTGTACGCTGCTTACGAA GTTATCTCTCTGATCGACGAATACGCTGCTTACACCTCTTCTACCGACGGTGACCTGG TTGGTCCGGGTCCGGGTCGTAAAGTTTCTCAGATCAAAATCGTTACCTCTCGTGTTA AAGCTGGTCCGGGTCCGGGTCCGCGTAAAGTTTCTCAGATCAAAATCGTTACCTCTC GTGTTAAAGGTCCGGGTCCGGGTCAGCCGCGTAAAGTTTCTCAGATCAAAATCGTTA CCTCTCGTGTTGGTCCGGGTCCGGGTCGTTCTCTGGTTCGTATCCGTAAAGTTCTGGA CTCTATCGCTGCTGGTCCGGGTCCGGGTGTTTCTCAGCAGCAGGCTATGGAATCTGT TTGCCACCCGATCATCGGTCCGGGTCCGGGTGACATCGTTTCTCAGCAGCAGGCTAT GGAATCTGTTTGCCACCCGGGTCCGGGTCCGGGTATCGTTTCTCAGCAGCAGGCTAT GGAATCTGTTTGCCACCCGATCGGTCCGGGTCCGGGTAACCGTTCTCTGGTTCGTAT CCGTAAAGTTCTGGACTCTATCGCTGGTCCGGGTCCGGGTTGCGCTTTCCAGGCTGC TATCTGCTCTCCGGCTTTCAAAGTTAAAGGTCCGGGTCCGGGTGACCAGCCGCGTAA AGTTTCTCAGATCAAAATCGTTACCTCTCGTGGTCCGGGTCCGGGTTTCAACCGTTCT CTGGTTCGTATCCGTAAAGTTCTGGACTCTATCGGTCCGGGTCCGGGTGGTTGCGCTT TCCAGGCTGCTATCTGCTCTCCGGCTTTCAAAGTTGGTCCGGGTCCGGGTAACTTCA ACCGTTCTCTGGTTCGTATCCGTAAAGTTCTGGACTCTGGTCCGGGTCCGGGTCGTG GTTGCGCTTTCCAGGCTGCTATCTGCTCTCCGGCTTTCAAAGGTCCGGGTCCGGGTTA CCGTGTTCTGACCACCAAAGAACTGGTTAAAGCTTTCACCCGTGGTCCGGGTCCGGG TACCCGTCTGTTCCTGGCTCAGAAAAA"

\section{Molecular docking of vaccine construct with TLR4}

A total of 29 models were generated from the molecular protein-protein docking between the vaccine construct and the TLR4 receptor using ClusPro 2.0 server. Only one model (000.23) with the lowest energy score (-1250.0 and highest binding affinity among other predicted docked complex fulfilled the desired criteria for best-docked complex and was eventually selected (Figure 7).

\section{Discussion}


313 Praziquantel (PZQ) is a pyrazinoisoquinole antihelminthic drug which is one of the few 314 commercially available anti-schistosoma drugs with few known side effects (Aragon et al. 2009).

315 The report of possible drug resistance to praziquantel due to drug pressure has now become of 316 huge public health concern. Several studies have reported remarkable cases of resistance to PZQ 317 which include the development of heritable trait that maintained drug resistance to PZQ in more 318 than six generations (Devaney 2006; Doenhoff et al. 2008; Fallon et al. 1996; Masamba et al. 319 2016; William et al. 2001). Only 13\% of targeted population received praziquantel treatment, 320 with the drug not capable of preventing reinfection, requires repeated treatment and its 321 characterised by reduction in efficiency among population with heavy infection (Tallima et al. 322 2017). With the endemicity of schistosomiasis still on the increase across many countries, with 323 increased cases of Schistosoma resistance to the most used and reliable drug PZQ. It is now 324 imperative to change the direction of treatment and focus on a lasting line of treatment by 325 targeting the parasitic lifecycle and its different developmental stages, which could well provide 326 a lasting solution to the spread of infection (Masamba et al. 2016). The current study focused on 327 retrieving seven (7) S. haematobium HSP sequences for the design of possible multi-epitope 328 subunit vaccine (logistically feasible and safely profiled as described by Shey et al. (2019)) 329 construct for the treatment of urinary schistosomiasis using computational approaches. Epitopes330 based vaccine will avoid responses against other unsuitable epitopes on antigen, thereby 331 generating more specific immune response against the antigen under consideration (Shey et al. 332 2019). Only three of these seven HSPs were eventually selected for the design of vaccine due to 333 their predicted high antigenic potential (Khatoon et al. 2017). Masamba et al. (2016) has 334 proposed that selective identification of small molecule inhibitors or peptides as an inhibitor of 335 HSPs at the schistosomula stage could be useful in targeting and preventing re-infection of schistosomiasis. Parasites use several mechanisms to upregulate Universal Stress Proteins 337 (including HSPs) which assist the parasite to tolerate different environmental and adverse conditions especially at the schistosomula stage of parasite development (Mbah et al. 2013). The HSPs CTL and HTL epitopes with predicted high immunogenicity were selected for inclusion in the vaccine construct. Thus the final vaccine construct had more of high affinities for MHC Class I, II and B-cell epitopes. The CTL-based response targets cells having intracellular viral, 342 bacterial or protozoan infection (Jordan \& Hunter 2010) while the HTL-based response is 343 essential for both humoral and cell-mediated immune response (Pross \& Lefkowitz 2008). 
344 Activated CD8+ cytotoxic T cells are reportedly able to promote death of parasite carrying host 345 MHC class I molecules in its surface (Zhou et al. 2012). Also mice immunized with $S$. 346 japonicum 22.6/26GST coupled to Sepharose 4B bead was able to reduce the parasite burden due 347 to an increase in the number of activated CD8+ cells (Zhou et al. 2012). Several studies have 348 used mouse model to understand host protective immune response against schistosome infections 349 (S. mansoni) and both antibodies and T cells are needed to ensure maximum protection (Colley 350 \& Secor 2014; Jankovic et al. 1999). Two previously reported spacer sequences (AAY and 351 GPGPG linkers) were used to improve the designed vaccine (Shey et al. 2019) by incorporating 352 between the predicted CTL and HTL epitopes respectively, thereby producing sequences with 353 minimal junctional immunogenicity leading to the design of a more potent multi-epitope vaccine 354 (Meza et al. 2017; Shey et al. 2019).

355 The allergenicity and antigenicity test of the designed vaccine predicted its safety for human use 356 and also revealed its potency to inhibit the entrance of Schistosomes into the human host (Ali et 357 al. 2017; Khatoon et al. 2017; Pandey et al. 2018). Using physiochemical parameters and 358 structural features, the designed vaccine weighed is $51 \mathrm{kDa}$, which is an average molecular 359 weight for a multi-subunit vaccine (Khatoon et al. 2017). This therefore favours the antigenicity 360 of the vaccine (Khatoon et al. 2017; Pandey et al. 2018). The theoretical pI of 9.11 showed that 361 the designed vaccine is basic in nature; the obtained higher aliphatic index score of 77.81 362 classified the vaccine protein as thermostable (Ali et al. 2017; Khatoon et al. 2017), while the 363 negative value of the Grand average of hydropathicity of the vaccine represents its hydrophilic 364 nature. The 3-dimensional structure obtained from the highly accurate homology modeling 365 servers (RaptorX and GalaxyRefine) contain sufficient information about the arrangement of 366 protein amino acid residues, percentage disorder and secondary structures. Also, the Ramachandran plot showed very few outliner residues with most of the residues clustering at very favourable regions thereby ratifying the quality of the overall model as satisfactory. This information was useful in the study of vaccine construct function, dynamics and interaction with 370 ligands and other proteins (Khatoon et al. 2017). To enhance the thermostability of the designed vaccine, novel disulphide bonds were further introduced into the multi-epitope subunit vaccine 372 protein (Rana \& Akhter 2016). In order to express the vaccine construct in the E. coli expression 373 system, an in silico cloning was done (Khatoon et al. 2017; Pandey et al. 2018). Codon 374 adaptation of the designed vaccine was done according to the usage of E. coli (strain K12) 
375

376

377

378

379

380

381

382

383

384

385

386

387

388

389

390

391

392

393

394

395

396

397

398

399

400

401

402

expression system using JCAT server and found the codon adaptive index to be 0.95 whereas, a score close to 1.0 was described elsewhere as satisfactory (Pandey et al. 2018). A protein-protein docking analysis was carried out to determine the immune response of TLR-4 agonists against the multi-epitope subunit vaccine designed by minimizing the potential energy of the complete system. The energy minimization ensured conformational stability of designed vaccine-TLR-4 complex by repairing the unnecessary geometry of the structure (Pandey et al. 2017; Pandey et al. 2015). El-Din (2016) has reported decrease in S. mansoni worm load, egg load and granuloma size during TLR4 and TLR 9 stimulation with upregulated expression of macrophages. TLR 4 is reportedly known to play significant role in the dentritic cells (DCs) and macropahges recognition of helminthes products; macrophages production of cytokines and development of Th2 responses (El-Din 2016; Kane et al. 2008). In a Th1/Th2 cytokine secretion assay and DNA microarray anaylysis, activated $\mathrm{T}$ cells, up regulation of some cytotoxic genes, followed by increase in parasite egg load have been reported in mice deficient in TLR (Zhang et al. 2011).

\section{Conclusion}

In this study, we used several immunoinformatics approaches to design vaccine thay may be effective against urinary schistosomiasis. Sequences of $S$. haematobium HSPs were retrieved from the database to design a multi-epitope subunit vaccine containing a CTL, HTL and BCL epitopes of varying length. The designed vaccine was stable with high antigenic properties, high binding affinity for TLR-4 receptor and was found to be non-allergic for human use. However, the designed vaccine requires experimental validation in order to establish it ability in controlling Schistosoma infection through the generation of effective immune response and memory.

\section{Competing Interest}

All authors declared no competing interest

\section{Funding Statement:}

The authors receive not funding for this work. 
404

405

406

407

408

409

410

411

412

413

414

415

416

417

418

419

420

421

422

423

424

425

426

427

428

429

430

431

432

433

434

435

436

437

438

439

440

441

442

443

444

445

446

447

448

449

450

Abdulkadir A, Ahmed M, Abubakar BM, Suleiman IE, Yusuf I, Imam IM, Sule AA, Tela UM, Dogo HM, Yakasai AM, and Musa BM. 2017. Prevalence of urinary schistosomiasis in Nigeria, 19942015: Systematic review and meta-analysis. African Journal of Urology 23:228-239. https://doi.org/10.1016/j.afju.2016.11.004

Adebayo AS, Suryavanshi MV, Bhute S, Agunloye AM, Isokpehi RD, Anumudu CI, and Shouche YS. 2017. The microbiome in urogenital schistosomiasis and induced bladder pathologies. PLoS neglected tropical diseases 11:e0005826-e005826. 10.1371/journal.pntd.0005826

Adenowo AF, Oyinloye BE, Ogunyinka BI, and Kappo AP. 2015. Impact of human schistosomiasis in sub-Saharan Africa. The Brazilian journal of infectious diseases : an official publication of the Brazilian Society of Infectious Diseases 19:196-205. 10.1016/j.bjid.2014.11.004

Ali M, Pandey RK, Khatoon N, Narula A, Mishra A, and Prajapati VK. 2017. Exploring dengue genome to construct a multi-epitope based subunit vaccine by utilizing immunoinformatics approach to battle against dengue infection. Scientific Reports 7:9232-9232. 10.1038/s41598-017-09199-w

Alsaqabi SM, and Lotfy WM. 2014. Praziquantel: A review. Veterinary Science \& Technology 5:1.

Aragon AD, Imani RA, Blackburn VR, Cupit PM, Melman SD, Goronga T, Webb T, Loker ES, and Cunningham C. 2009. Towards an understanding of the mechanism of action of praziquantel. Molecular and biochemical parasitology 164:57-65. 10.1016/j.molbiopara.2008.11.007

Barsoum RS. 2013. Urinary schistosomiasis: review. Journal of advanced research 4:453-459. 10.1016/j.jare.2012.08.004

Bergquist R. 2013. Closing in on 'perhaps the most dreadful of the remaining plagues': an independent view of the multidisciplinary alliance to optimize schistosomiasis control in Africa. Acta tropica 128:179-181. 10.1016/j.actatropica.2013.08.016

Braschi S, Curwen RS, Ashton PD, Verjovski-Almeida S, and Wilson A. 2006. The tegument surface membranes of the human blood parasite Schistosoma mansoni: A proteomic analysis after differential extraction. Proteomics 6:1471-1482. 10.1002/pmic.200500368

Chevalier FD, Le Clec'h W, Eng N, Rugel AR, Assis RRd, Oliveira G, Holloway SP, Cao X, Hart PJ, LoVerde PT, and Anderson TJC. 2016. Independent origins of loss-of-function mutations conferring oxamniquine resistance in a Brazilian schistosome population. International journal for parasitology 46:417-424. 10.1016/j.ijpara.2016.03.006

Cioli D, Pica-Mattoccia L, Basso A, and Guidi A. 2014. Schistosomiasis control: praziquantel forever? Molecular and biochemical parasitology 195:23-29. 10.1016/j.molbiopara.2014.06.002

Colley DG, and Secor WE. 2014. Immunology of human schistosomiasis. Parasite immunology 36:347357. 10.1111/pim.12087

Couto FFB, Coelho PMZ, Araújo N, Kusel JR, Katz N, Jannotti-Passos LK, and Mattos ACA. 2011. Schistosoma mansoni: a method for inducing resistance to praziquantel using infected Biomphalaria glabrata snails. Memorias do Instituto Oswaldo Cruz 106:153-157. 10.1590/s007402762011000200006

Das JK, Xiong X, Ren X, Yang J-M, and Song J. 2019. Heat Shock Proteins in Cancer Immunotherapy. Journal of Oncology 2019:9. 10.1155/2019/3267207

Devaney E. 2006. Thermoregulation in the life cycle of nematodes. International journal for parasitology 36:641-649. 10.1016/j.ijpara.2006.02.006

Doenhoff MJ, Cioli D, and Utzinger J. 2008. Praziquantel: mechanisms of action, resistance and new derivatives for schistosomiasis. Current opinion in infectious diseases 21:659-667. 10.1097/QCO.0b013e328318978f

El-Din SAS. 2016. Role of Toll Like Receptors 4, 5 and 9 Ligands in Pathogenesis and

Outcome of Intestinal and Hepatic Schistosomiaisis Caused by

Schistosoma mansoni. Research Journal of Parasitology 11:1-12.

Peer) reviewing PDF | (2019:10:42418:2:1:NEW 15 Feb 2020) 
451

452

453

454

455

456

457

458

459

460

461

462

463

464

465

466

467

468

469

470

471

472

473

474

475

476

477

478

479

480

481

482

483

484

485

486

487

488

489

490

491

492

493

494

495

496

497

498

499

500

El-Manzalawy Y, Dobbs D, and Honavar V. 2008. Predicting linear B-cell epitopes using string kernels. Journal of molecular recognition : JMR 21:243-255. 10.1002/jmr.893

Fallon PG, Tao LF, Ismail MM, and Bennett JL. 1996. Schistosome resistance to praziquantel: Fact or artifact? Parasitology today (Personal ed) 12:316-320. 10.1016/0169-4758(96)10029-6

Heo L, Park H, and Seok C. 2013. GalaxyRefine: Protein structure refinement driven by side-chain repacking. Nucleic Acids Research 41:W384-W388. 10.1093/nar/gkt458

Ishida K, and Jolly ER. 2016. Hsp70 May Be a Molecular Regulator of Schistosome Host Invasion. PLoS neglected tropical diseases 10:e004986-e0004986. 10.1371/journal.pntd.0004986

Jankovic D, Wynn TA, Kullberg MC, Hieny S, Caspar P, James S, Cheever AW, and Sher A. 1999. Optimal vaccination against Schistosoma mansoni requires the induction of both B cell- and IFNgamma-dependent effector mechanisms. Journal of immunology (Baltimore, Md : 1950) 162:345351.

Jordan KA, and Hunter CA. 2010. Regulation of CD8+ T cell responses to infection with parasitic protozoa. Experimental parasitology 126:318-325. 10.1016/j.exppara.2010.05.008

Kane CM, Jung E, and Pearce EJ. 2008. Schistosoma mansoni egg antigen-mediated modulation of Tolllike receptor (TLR)-induced activation occurs independently of TLR2, TLR4, and MyD88. Infection and immunity 76:5754-5759. 10.1128/IAI.00497-08

Khatoon N, Pandey RK, and Prajapati VK. 2017. Exploring Leishmania secretory proteins to design B and T cell multi-epitope subunit vaccine using immunoinformatics approach. Scientific Reports 7:8285.

Kozakov D, Hall DR, Xia B, Porter KA, Padhorny D, Yueh C, Beglov D, and Vajda S. 2017. The ClusPro web server for protein-protein docking. Nature Protocols 12:255-278. 10.1038/nprot.2016.169

Lee SJ, Shin SJ, Lee MH, Lee M-G, Kang TH, Park WS, Soh BY, Park JH, Shin YK, Kim HW, Yun CH, Jung ID, and Park Y-M. 2014. A potential protein adjuvant derived from Mycobacterium tuberculosis Rv0652 enhances dendritic cells-based tumor immunotherapy. PLoS One 9:e104351e104351. 10.1371/journal.pone.0104351

Masamba P, Adenowo AF, Oyinloye BE, and Kappo AP. 2016. Universal Stress Proteins as New Targets for Environmental and Therapeutic Interventions of Schistosomiasis. International journal of environmental research and public health 13:972. 10.3390/ijerph13100972

Mbah AN, Mahmud O, Awofolu OR, and Isokpehi RD. 2013. Inferences on the biochemical and environmental regulation of universal stress proteins from Schistosomiasis parasites. Advances and applications in bioinformatics and chemistry: AABC 6:15-27. 10.2147/AABC.S37191

McNulty S, Colaco CA, Blandford LE, Bailey CR, Baschieri S, and Todryk S. 2013. Heat-shock proteins as dendritic cell-targeting vaccines--getting warmer. Immunology 139:407-415. 10.1111/imm.12104

Melman SD, Steinauer ML, Cunningham C, Kubatko LS, Mwangi IN, Wynn NB, Mutuku MW, Karanja DMS, Colley DG, Black CL, Secor WE, Mkoji GM, and Loker ES. 2009. Reduced susceptibility to praziquantel among naturally occurring Kenyan isolates of Schistosoma mansoni. PLoS neglected tropical diseases 3:e504-e504. 10.1371/journal.pntd.0000504

Meza B, Ascencio F, Sierra-Beltrán AP, Torres J, and Angulo C. 2017. A novel design of a multiantigenic, multistage and multi-epitope vaccine against Helicobacter pylori: An in silico approach. Infection, genetics and evolution : journal of molecular epidemiology and evolutionary genetics in infectious diseases 49:309-317. 10.1016/j.meegid.2017.02.007

Milesi M, Indovina C, Dino O, Di Bella F, Di Lorenzo F, Sanfilippo A, Di Bernardo F, Sodano C, Prestileo T, and Immigrant Take Care Advocacy team P. 2019. Urinary schistosomiasis in migrant population: a case series from a single centre in southern Italy. Infection 47:395-398. 10.1007/s15010-018-1244-z

Onile OS. 2014. Relationship between Physico-chemical Parameters and Phylogenetics Study of Human Low Density Lipoprotein Receptor-Related Protein (LRP). British Biotechnology Journal 4:289.

Peer) reviewing PDF | (2019:10:42418:2:1:NEW 15 Feb 2020) 
501

502

503

504

505

506

507

508

509

510

511

512

513

514

515

516

517

518

519

520

521

522

523

524

525

526

527

528

529

530

531

532

533

534

535

536

537

538

539

540

541

542

543

544

545

546

547

548

549

550

551

Onile OS, Awobode HO, Oladele VS, Agunloye AM, and Anumudu CI. 2016. Detection of Urinary Tract Pathology in Some Schistosoma haematobium Infected Nigerian Adults. Journal of tropical medicine 2016:5405207-5405207. 10.1155/2016/5405207

Onile OS, Calder B, Soares NC, Anumudu CI, and Blackburn JM. 2017. Quantitative label-free proteomic analysis of human urine to identify novel candidate protein biomarkers for schistosomiasis. PLoS neglected tropical diseases 11:e0006045-e0006045. 10.1371/journal.pntd.0006045

Pandey RK, Bhatt TK, and Prajapati VK. 2018. Novel immunoinformatics approaches to design multiepitope subunit vaccine for malaria by investigating anopheles salivary protein. Scientific Reports 8:1125.

Pandey RK, Kumbhar BV, Srivastava S, Malik R, Sundar S, Kunwar A, and Prajapati VK. 2017. Febrifugine analogues as Leishmania donovani trypanothione reductase inhibitors: binding energy analysis assisted by molecular docking, ADMET and molecular dynamics simulation. Journal of biomolecular structure \& dynamics 35:141-158. 10.1080/07391102.2015.1135298

Pandey RK, Sharma D, Bhatt TK, Sundar S, and Prajapati VK. 2015. Developing imidazole analogues as potential inhibitor for Leishmania donovani trypanothione reductase: virtual screening, molecular docking, dynamics and ADMET approach. Journal of biomolecular structure \& dynamics 33:2541-2553. 10.1080/07391102.2015.1085904

Poddighe D, Castelli L, Pulcrano G, Grosini A, Balzaretti M, Spadaro S, and Bruni P. 2016. Urinary Schistosomiasis in an Adolescent Refugee from Africa: An Uncommon Cause of Hematuria and an Emerging Infectious Disease in Europe. Journal of immigrant and minority health 18:12371240. 10.1007/s10903-015-0272-3

Pross S, and Lefkowitz D. 2008. in xPharm: The Comprehensive Pharmacology Reference. Elsevier:1-4.

Rana A, and Akhter Y. 2016. A multi-subunit based, thermodynamically stable model vaccine using combined immunoinformatics and protein structure based approach. Immunobiology 221:544557.

Rollinson D, Knopp S, Levitz S, Stothard JR, Tchuem Tchuenté L-A, Garba A, Mohammed KA, Schur N, Person B, Colley DG, and Utzinger J. 2013. Time to set the agenda for schistosomiasis elimination. Acta tropica 128:423-440. 10.1016/j.actatropica.2012.04.013

Shanmugam A, Rajoria S, George AL, Mittelman A, Suriano R, and Tiwari RK. 2012. Synthetic Toll like receptor-4 (TLR-4) agonist peptides as a novel class of adjuvants. PLoS One 7:e30839-e30839. 10.1371/journal.pone.0030839

Shey RA, Ghogomu SM, Esoh KK, Nebangwa ND, Shintouo CM, Nongley NF, Asa BF, Ngale FN, Vanhamme L, and Souopgui J. 2019. In-silico design of a multi-epitope vaccine candidate against onchocerciasis and related filarial diseases. Scientific Reports 9:4409-4409. 10.1038/s41598-01940833-x

Tallima H, Dvořák J, Kareem S, Abou El Dahab M, Abdel Aziz N, Dalton JP, and El Ridi R. 2017. Protective immune responses against Schistosoma mansoni infection by immunization with functionally active gut-derived cysteine peptidases alone and in combination with glyceraldehyde 3-phosphate dehydrogenase. PLoS neglected tropical diseases 11:e0005443-e0005443. 10.1371/journal.pntd.0005443

Uysal HK, Akgul O, Aliyev E, Tunc MH, and Oner YA. 2014. A rare case of urinary schistosomiasis in Turkey. Travel medicine and infectious disease 12:283-285. 10.1016/j.tmaid.2013.11.002

Venugopal PG, Nutman TB, and Semnani RT. 2009. Activation and regulation of toll-like receptors (TLRs) by helminth parasites. Immunologic research 43:252-263. 10.1007/s12026-008-8079-0

William S, Botros S, Ismail M, Farghally A, Day TA, and Bennett JL. 2001. Praziquantel-induced tegumental damage in vitro is diminished in schistosomes derived from praziquantel-resistant infections. Parasitology 122 Pt 1:63-66. 10.1017/s0031182000007137

Zhang M, Gao Y, Du X, Zhang D, Ji M, and Wu G. 2011. Toll-like receptor (TLR) 2 and TLR4 deficiencies exert differential in vivo effects against Schistosoma japonicum. Parasite immunology 33:199-209. 10.1111/j.1365-3024.2010.01265.x

Peer] reviewing PDF | (2019:10:42418:2:1:NEW 15 Feb 2020) 
552 Zhou Y, Zhang H, Sun X-J, Zheng D, Liang Y-J, Luo J, Wang Y, and Zhang Z-S. 2012. Murine CD8(+)T cell cytotoxicity against schistosomula induced by inoculation of schistosomal 22.6/26GST coupled Sepharose 4B beads. Vaccine 30:2440-2447. 10.1016/j.vaccine.2012.01.068

\section{Data Availability}

556 All data generated or analysed during this study are included in this published article

\section{Authors Contributions:}

559 OOS conceived of the study, participated in its design and analysis of data, helped in the draft of 560 manuscript; FAI also participated in the design of the study, draft of manuscript and was 561 involved in codon adaptation and in silico cloning. AAA was involved the sequence retrieval, 562 prediction of CTL and HTL epitopes, draft of manuscript and construction of subunit vaccine; 563 ACI and OBF participated in design of study, draft and correction of manuscript. All authors 564 read and approved the final manuscript 
Figure 1

Diagram of final vaccine construct.

The multi-epitope vaccine sequence consisting of 493 amino acid residues; of which the adjuvant and CTL epitope has been joined by EAAK linker, where AAY and GPGPG linkers were used to join the CTL and HTL epitopes respectively 
A

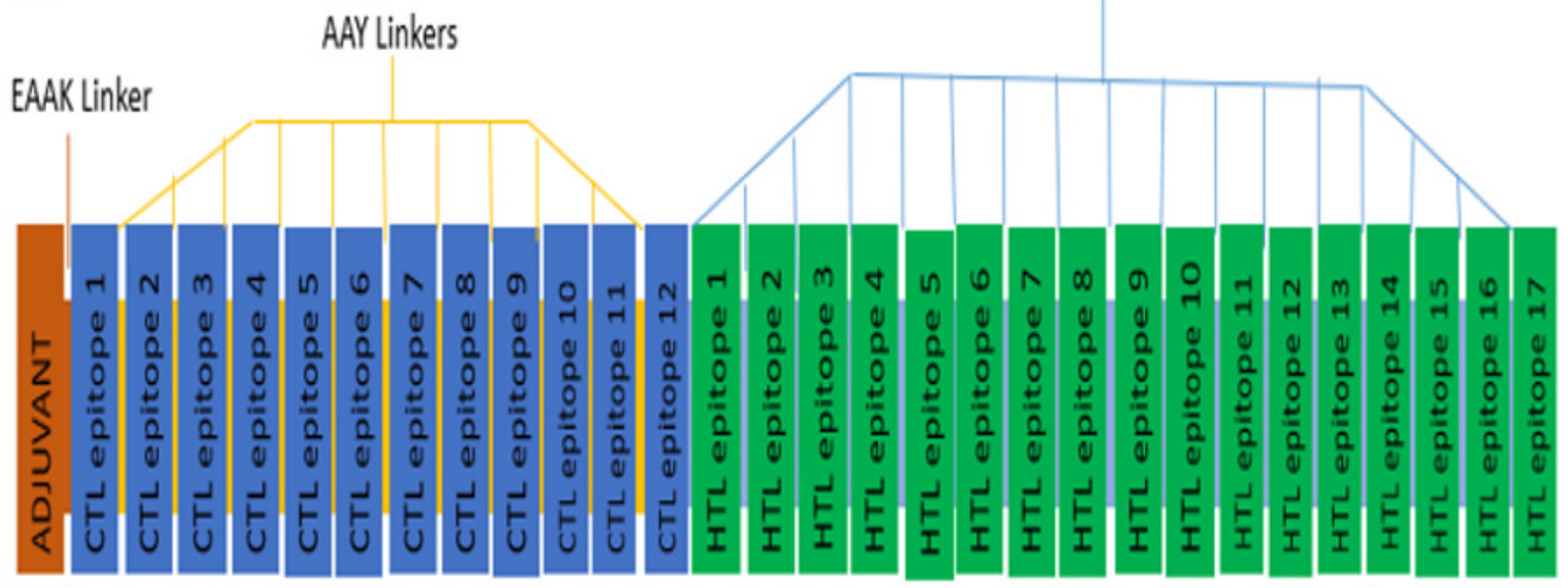

B

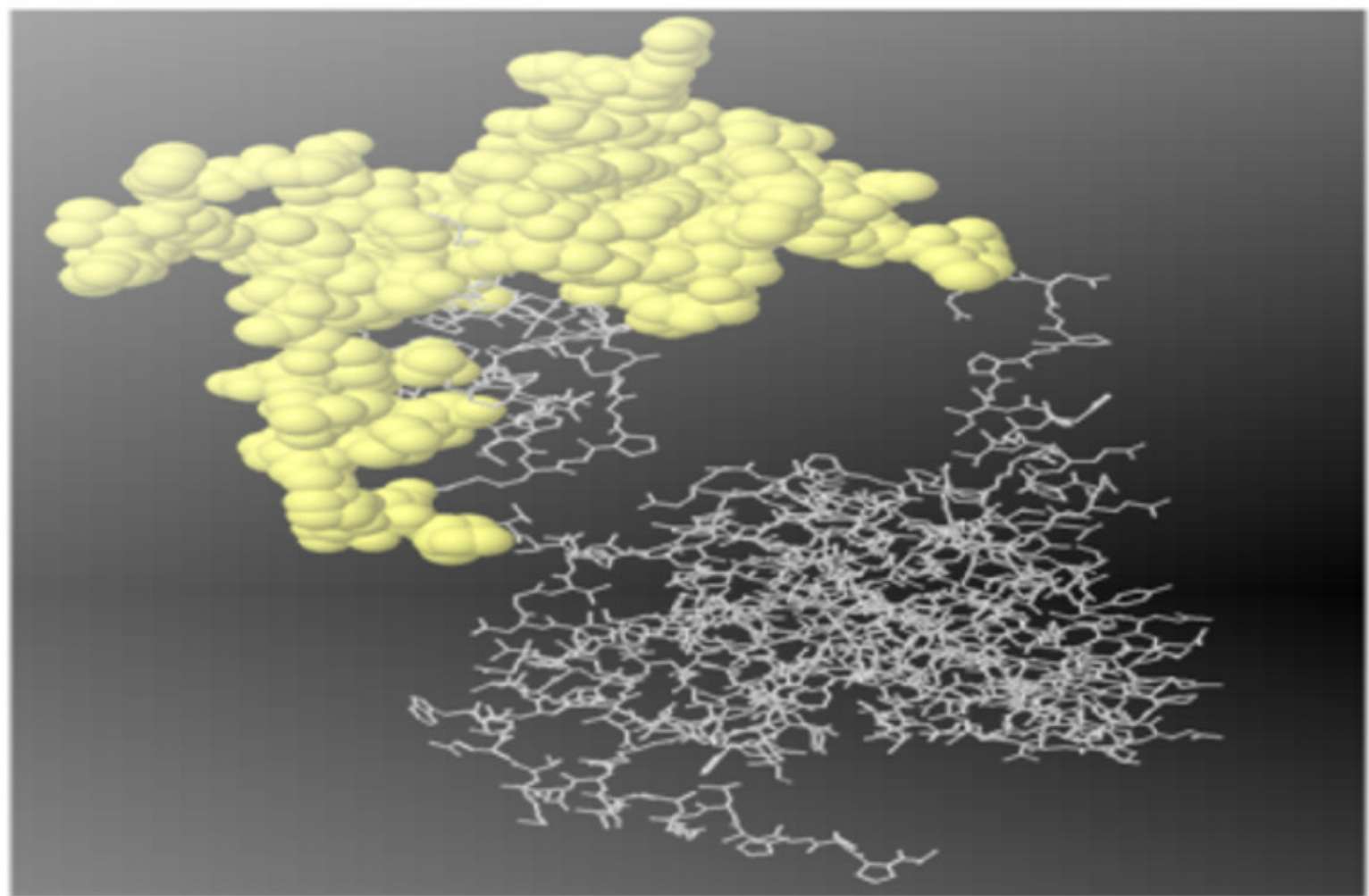


Figure 2

Tertiary structure of predicted vaccine construct showing the helix, sheet and coiled region

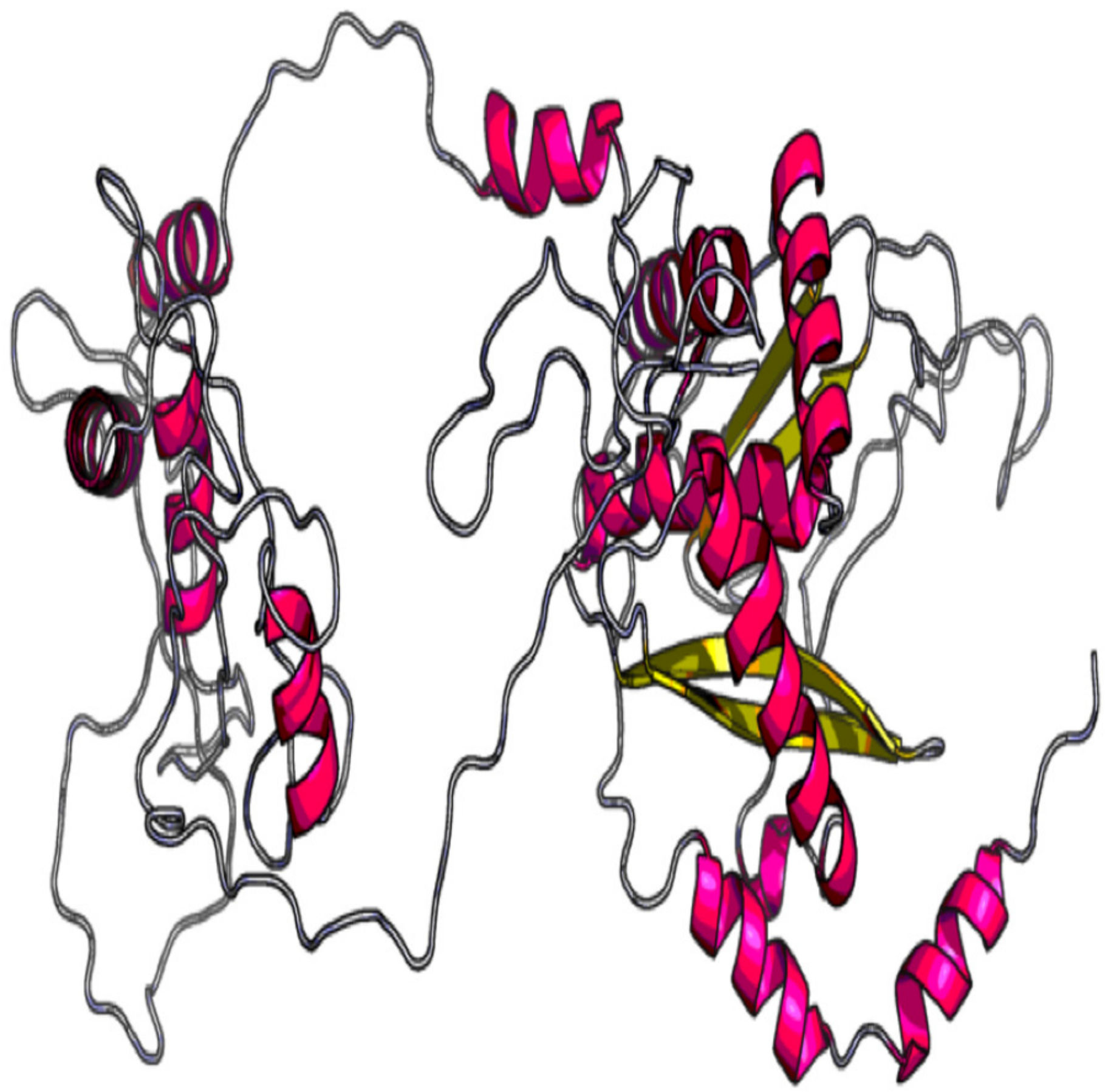


Figure 3

PROSA validation of 3D vaccine structure showing Z-score (-6.01)

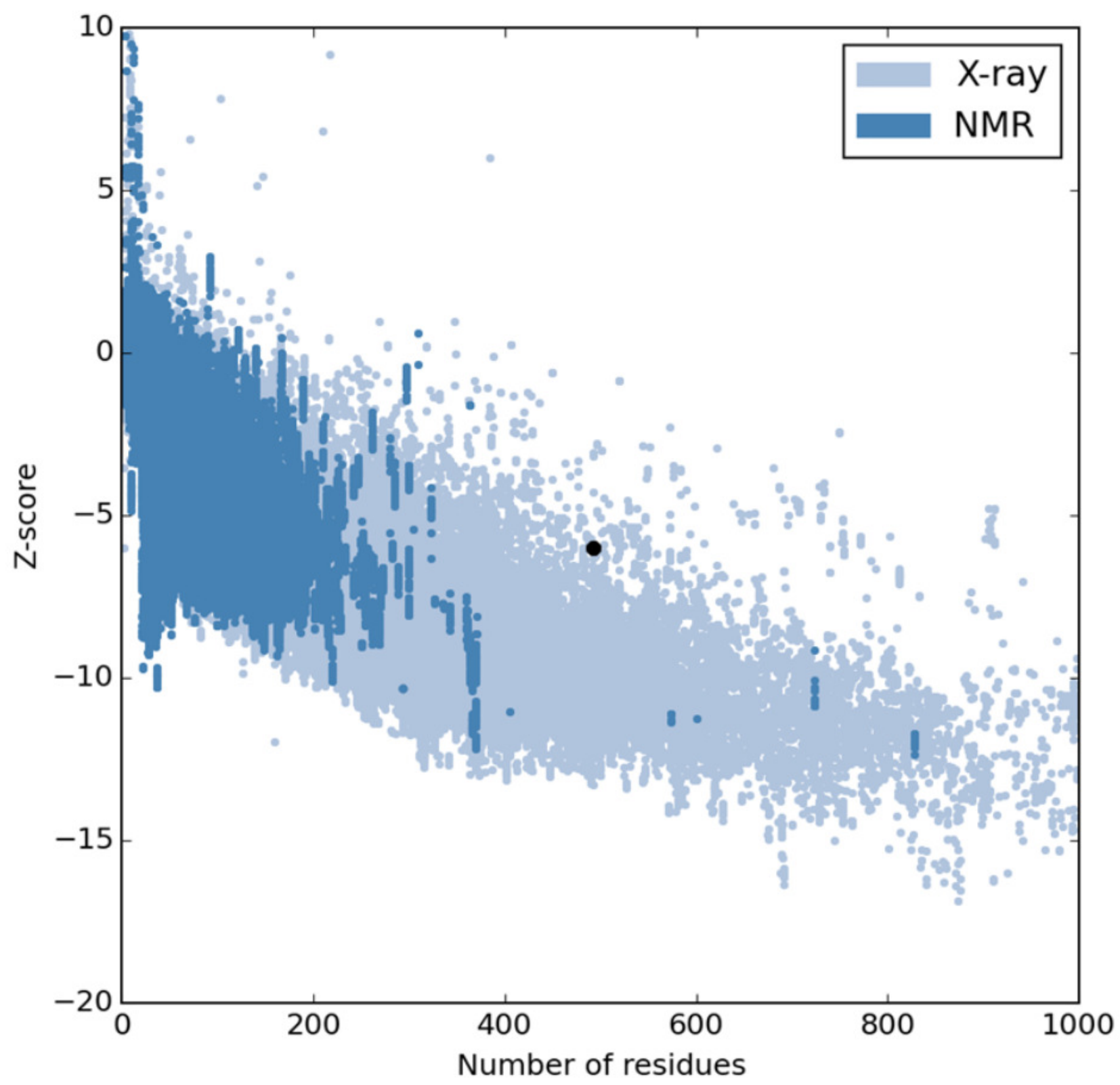


Figure 4

Ramachandran plot formation to the validated 3D modelled structure of vaccine protein.

The plot showed $93.3 \%$ residues of vaccine protein in favoured region

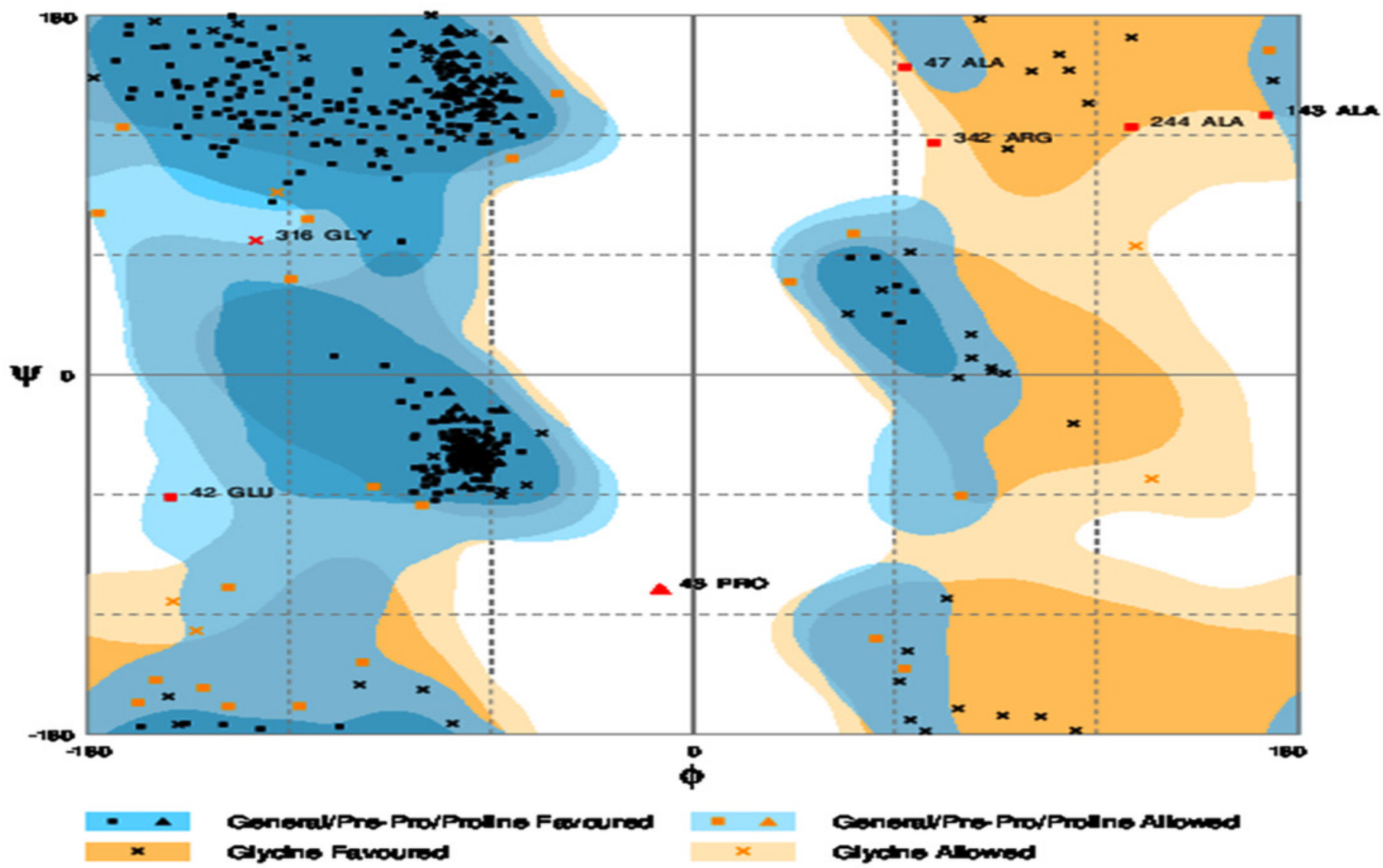


Figure 5

Mutated and stabilized vaccine construct protein by disulphide engineering.

The engineered protein showed the disulfide bonds in red colour as presented by the mutated platform of Disulfide by Design server. 


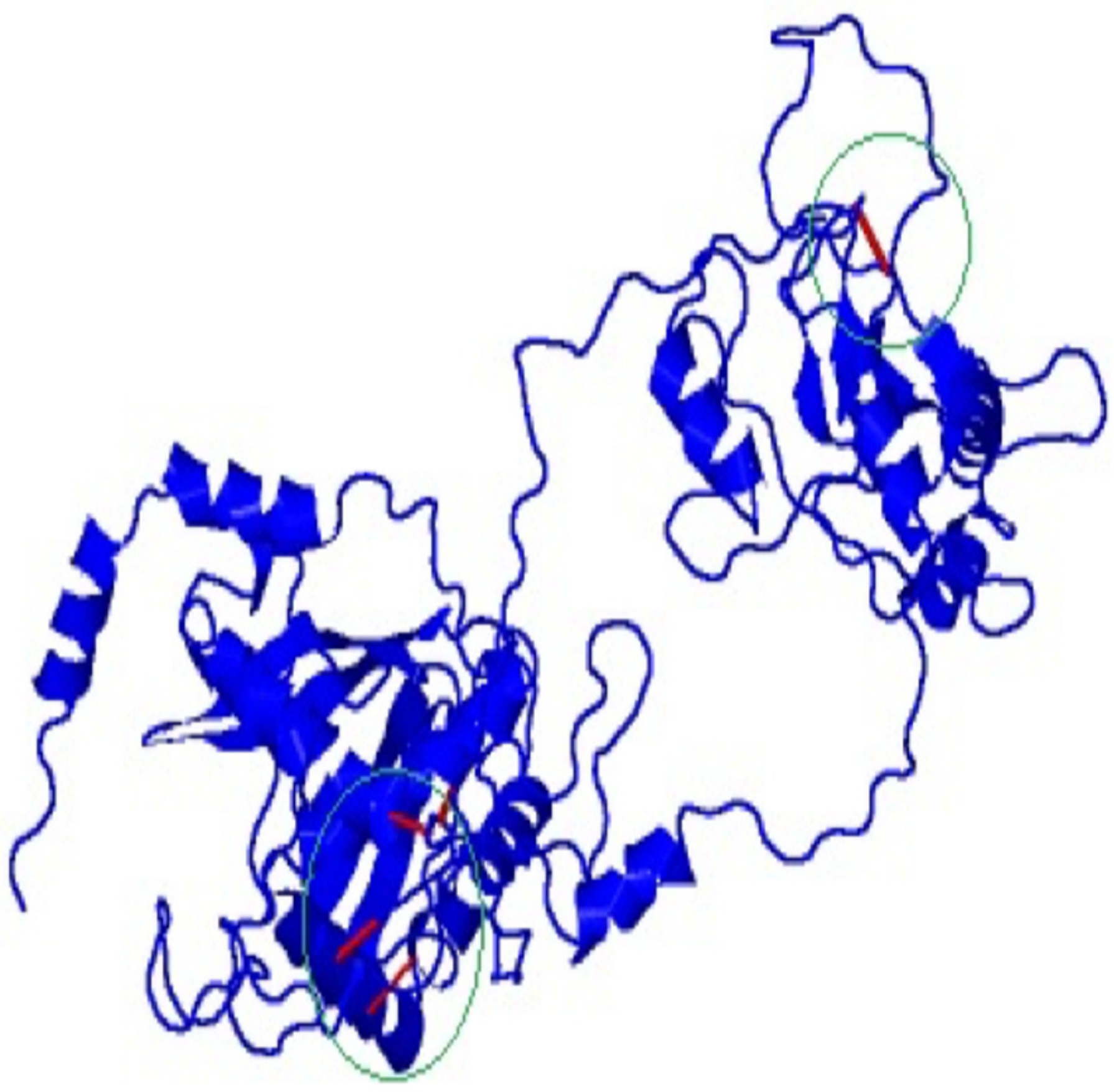


Figure 6

In silico restriction cloning for adapted vaccine construct into $\mathrm{pET} 28 \mathrm{a}(+)$ expression vector.

The adapted vaccine sequences is shown in red colour as the region of insert while the black circle represent the vector. 


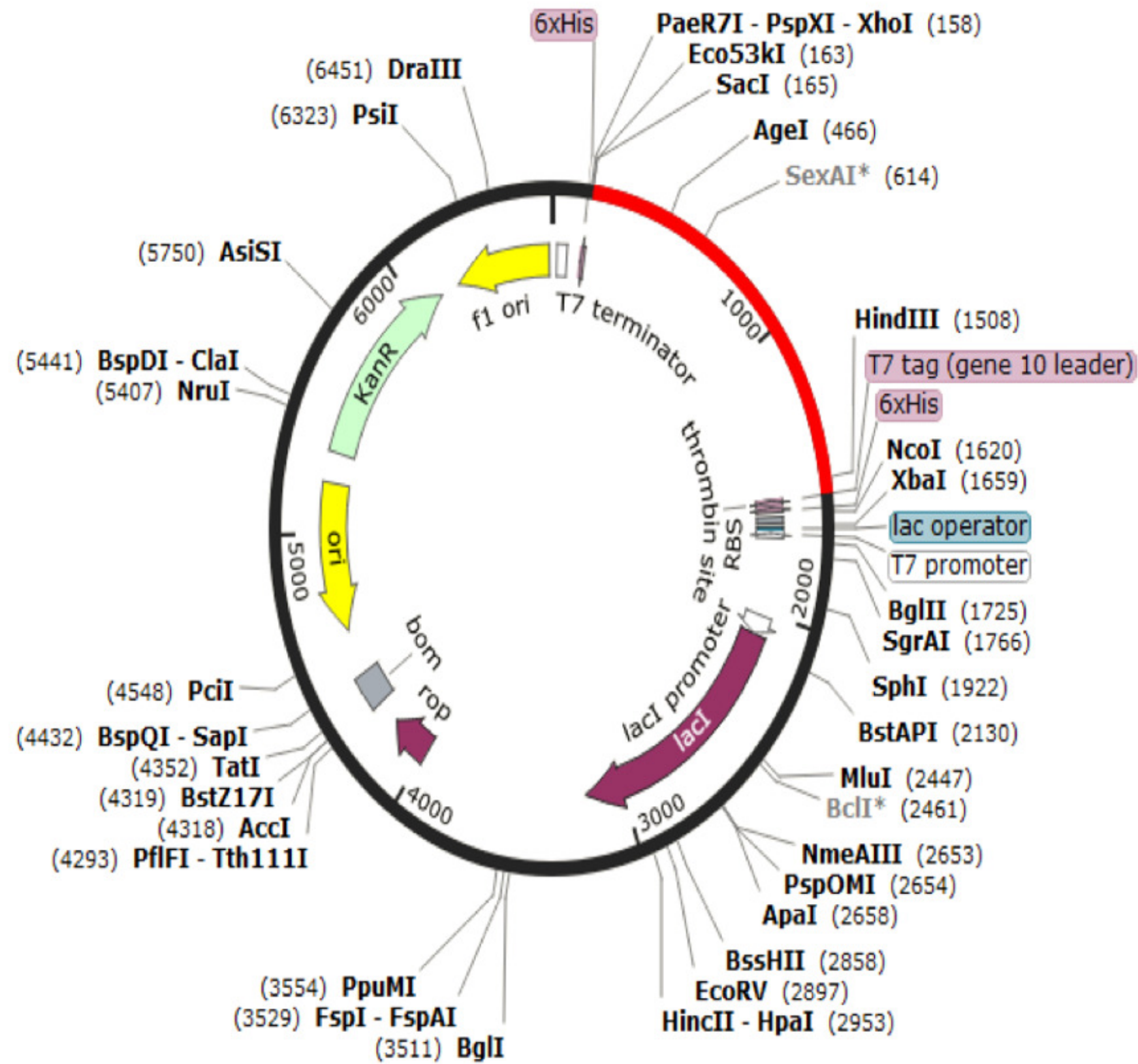

\section{S. haematobium_Vaccine $6693 \mathrm{bp}$}




\section{Figure 7}

The docked complex showing the vaccine protein (dot) and TLR-4 receptor (cartoon) interaction.

\section{Vaccine Construct}

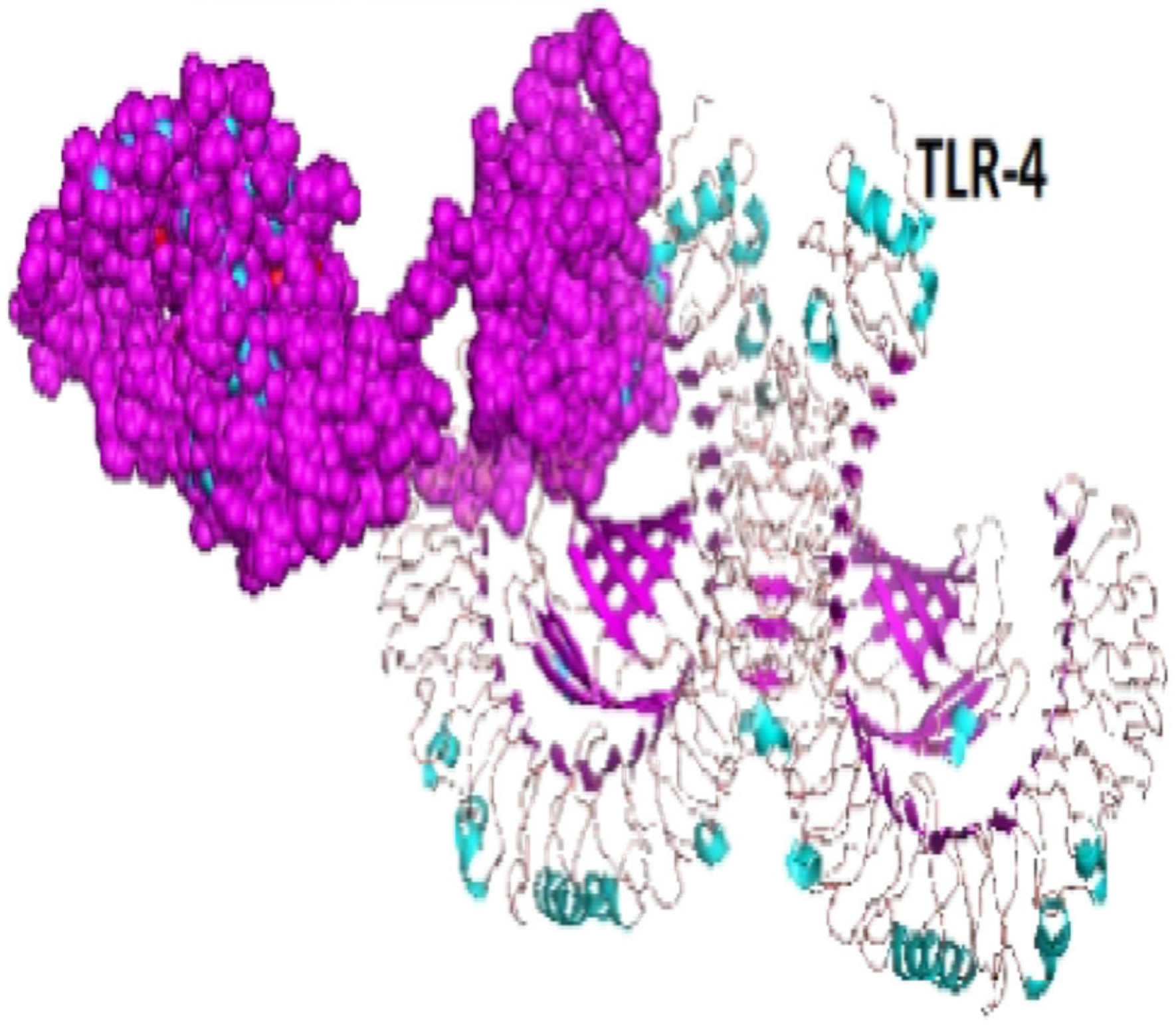




\section{Table $\mathbf{1}$ (on next page)}

Retrieved S. haematobium Heat shock protein accession numbers from the NCBI database and their antigenicity scores 


\begin{tabular}{|c|c|c|c|c|}
\hline $\begin{array}{l}\text { Serial } \\
\text { No. }\end{array}$ & $\begin{array}{l}\text { Protein } \\
\text { Accession No. }\end{array}$ & Protein Name & $\begin{array}{l}\text { Antigenicity } \\
\text { Scores }\end{array}$ & $\begin{array}{l}\text { Selected/ } \\
\text { Non-selected }\end{array}$ \\
\hline 1 & KGB40455.1 & $60 \mathrm{kDa}$ Heat shock protein & 0.669986 & Non-selected \\
\hline 2 & KGB38609.1 & $75 \mathrm{kDa}$ Heat shock protein & 0.665038 & Non-selected \\
\hline 3 & KGB41273.1 & $10 \mathrm{kDa}$ Heat shock protein & 0.608315 & Non-selected \\
\hline 4 & KGB37622.1 & Heat shock protein 83 & 0.665191 & Non-selected \\
\hline 5 & KGB34481.1 & $97 \mathrm{kDa}$ Heat shock protein & 0.926815 & Selected \\
\hline 6 & XP_012799478.1 & Heat shock protein beta- 11 & 0.895751 & Selected \\
\hline 7 & KGB37337.1 & $\begin{array}{l}\text { Activator of } 90 \mathrm{kDa} \text { Heat } \\
\text { shock protein ATPase } 1\end{array}$ & 0.858776 & Selected \\
\hline
\end{tabular}




\section{Table 2 (on next page)}

Predicted cytotoxic T-lymphocyte (CTL) epitopes and their immunogenicity scores for $S$. haematobium as obtained from the immune epitope database 
1

\begin{tabular}{|c|c|c|c|c|c|c|}
\hline $\mathbf{S} / \mathbf{N}$ & Accession ID & Epitopes & Scores & Lenght & $\begin{array}{l}\text { Immunogenicity } \\
\text { Scores }\end{array}$ & $\begin{array}{ll}\text { Selected/ Non } \\
\text { selected }\end{array}$ \\
\hline \multirow[t]{11}{*}{1} & KGB34481.1 & LDMTEEWLY & 0.7760 & 9 & 0.35427 & Selected \\
\hline & & QTEEIDGTL & 0.9769 & 9 & 0.33501 & Selected \\
\hline & & SIAAGEPTY & 0.8120 & 9 & 0.19264 & Selected \\
\hline & & SQAQLIEEY & 1.3793 & 9 & 0.15362 & Selected \\
\hline & & NSKNAVEEY & 1.7785 & 9 & 0.14345 & Selected \\
\hline & & FTVIEQCLY & 2.7477 & 9 & 0.0838 & Selected \\
\hline & & QLEDMIVQY & 1.8465 & 9 & -0.02374 & Non-selected \\
\hline & & VTDIVSQQQ & 0.8464 & 9 & -0.13985 & Non-selected \\
\hline & & FTEPRKIKL & 0.7619 & 9 & -0.14494 & Non-selected \\
\hline & & FTTKQLNEF & 1.0480 & 9 & -0.2746 & Non-selected \\
\hline & & SIEVSNMQF & 0.8128 & 9 & -0.30903 & Non-selected \\
\hline 2 & KGB39720.1 & GSDGCFVSF & 2.2740 & 9 & 0.03718 & Selected \\
\hline \multirow[t]{12}{*}{3} & XP_012797099. & FSGNITGIF & 1.0475 & 9 & 0.27699 & Selected \\
\hline & 1 & & & & & \\
\hline & & KLDGEANVY & 2.2518 & 9 & 0.19429 & Selected \\
\hline & & GSKIENDLY & 2.0995 & 9 & 0.16757 & Selected \\
\hline & & EVISLIDEY & 0.7906 & 9 & 0.06843 & Selected \\
\hline & & TSSTDGDLV & 0.9172 & 9 & 0.0511 & Selected \\
\hline & & WSDKDATGW & 1.0050 & 9 & -0.09881 & Non-selected \\
\hline & & YITLLKEDY & 1.1013 & 9 & -0.1149 & Non-selected \\
\hline & & WTSSTDGDL & 0.9051 & 9 & -0.11993 & Non-selected \\
\hline & & LAQKNVPAY & 0.9310 & 9 & -0.20854 & Non-selected \\
\hline & & FLAALKQTY & 1.7104 & 9 & -0.23681 & Non-selected \\
\hline & & AALKQTYGY & 0.7829 & 9 & -0.28018 & Non-selected \\
\hline
\end{tabular}

2 *Only epitopes with Comb score $>0.75$ were selected for immunogenicity test and CTL epitopes (12)

3 with high immunogenicity score (+ve ) were selected and subjected to the vaccine designing

4 
Table 3 (on next page)

A) Predicted Helper T-lymphocyte (HTL) epitopes and their percentile ranks for $S$. haematobium as obtained from the immune epitope database B) Interferon Gamma (IFN) Inducing capacity test for the predicted HTL epitopes for urinary schistosomiasis 


\begin{tabular}{|c|c|c|c|c|c|c|}
\hline AAllele & Seq. No. & Start & End & Epitope & Method & $\begin{array}{l}\text { Percentile } \\
\text { Rank* }\end{array}$ \\
\hline H2-IAd & 2 & 54 & 68 & RKVSQIKIVTSRVKA & Consensus (smm/nn) & 0.38 \\
\hline H2-IAd & 2 & 53 & 67 & PRKVSQIKIVTSRVK & Consensus (smm/nn) & 0.47 \\
\hline H2-IAd & 2 & 52 & 66 & QPRKVSQIKIVTSRV & Consensus (smm/nn) & 0.48 \\
\hline H2-IAd & 3 & 435 & 449 & RSLVRIRKVLDSIAA & Consensus (smm/nn) & 0.64 \\
\hline H2-IAd & 3 & 500 & 514 & VSQQQAMESVCHPII & Consensus (smm/nn) & 0.91 \\
\hline H2-IAd & 3 & 498 & 512 & DIVSQQQAMESVCHP & Consensus (smm/nn) & 0.94 \\
\hline H2-IAd & 3 & 499 & 513 & IVSQQQAMESVCHPI & Consensus (smm/nn) & 1.03 \\
\hline H2-IAd & 3 & 434 & 448 & NRSLVRIRKVLDSIA & Consensus (smm/nn) & 1.04 \\
\hline H2-IAd & 3 & 100 & 114 & CAFQAAICSPAFKVK & Consensus (smm/nn) & 1.05 \\
\hline H2-IAd & 2 & 51 & 65 & DQPRKVSQIKIVTSR & Consensus (smm/nn) & 1.12 \\
\hline H2-IAd & 3 & 433 & 447 & FNRSLVRIRKVLDSI & Consensus (smm/nn) & 1.21 \\
\hline H2-IAd & 3 & 99 & 113 & GCAFQAAICSPAFKV & Consensus (smm/nn) & 1.23 \\
\hline H2-IAd & 3 & 432 & 446 & NFNRSLVRIRKVLDS & Consensus (smm/nn) & 1.27 \\
\hline H2-IAd & 3 & 98 & 112 & RGCAFQAAICSPAFK & Consensus (smm/nn) & 1.32 \\
\hline H2-IAd & 1 & 214 & 228 & YRVLTTKELVKAFTR & Consensus (smm/nn) & 1.35 \\
\hline H2-IAd & 1 & 289 & 303 & TRLFLAQKNVPAYDL & Consensus (smm/nn) & 1.37 \\
\hline H2-IAd & 3 & 497 & 511 & TDIVSQQQAMESVCH & Consensus (smm/nn) & 1.4 \\
\hline${ }^{\text {BSerial No. }}$ & \multicolumn{3}{|c|}{ Epitope } & \begin{tabular}{l|l|} 
& Method \\
\end{tabular} & \begin{tabular}{l|l} 
& Result \\
\end{tabular} & Score \\
\hline Epitope_1 & \multicolumn{3}{|c|}{ RKVSQIKIVTSRVKA } & MERCI & POSITIVE & 1 \\
\hline Epitope 2 & \multicolumn{3}{|c|}{ PRKVSQIKIVTSRVK } & MERCI & POSITIVE & 1 \\
\hline Epitope 3 & \multicolumn{3}{|c|}{ QPRKVSQIKIVTSRV } & SVM & POSITIVE & 0.57919869 \\
\hline Epitope_4 & \multicolumn{3}{|c|}{ RSLVRIRKVLDSIAA } & MERCI & POSITIVE & 6 \\
\hline Epitope 5 & \multicolumn{3}{|c|}{ VSQQQAMESVCHPII } & MERCI & POSITIVE & 1 \\
\hline Epitope 6 & \multicolumn{3}{|c|}{ DIVSQQQAMESVCHP } & MERCI & POSITIVE & 1 \\
\hline Epitope_7 & \multicolumn{3}{|c|}{ IVSQQQAMESVCHPI } & MERCI & POSITIVE & 1 \\
\hline Epitope 8 & \multicolumn{3}{|c|}{ NRSLVRIRKVLDSIA } & MERCI & POSITIVE & 8 \\
\hline Epitope 9 & \multicolumn{3}{|c|}{ CAFQAAICSPAFKVK } & MERCI & POSITIVE & 1 \\
\hline Epitope_10 & \multicolumn{3}{|c|}{ DQPRKVSQIKIVTSR } & SVM & POSITIVE & 0.56096058 \\
\hline Epitope 11 & \multicolumn{3}{|c|}{ FNRSLVRIRKVLDSI } & MERCI & POSITIVE & 9 \\
\hline Epitope_12 & \multicolumn{3}{|c|}{ GCAFQAAICSPAFKV } & MERCI & POSITIVE & 1 \\
\hline Epitope_13 & \multicolumn{3}{|c|}{ NFNRSLVRIRKVLDS } & MERCI & POSITIVE & 8 \\
\hline Epitope 14 & \multicolumn{3}{|c|}{ RGCAFQAAICSPAFK } & MERCI & POSITIVE & 2 \\
\hline Epitope_15 & \multicolumn{3}{|c|}{ YRVLTTKELVKAFTR } & MERCI & POSITIVE & 11 \\
\hline Epitope_16 & \multicolumn{3}{|c|}{ TRLFLAQKNVPAYDL } & MERCI & POSITIVE & 1 \\
\hline Epitope 17 & \multicolumn{3}{|c|}{ TDIVSQQQAMESVCH } & SVM & POSITIVE & 0.50508595 \\
\hline
\end{tabular}

2 *Only HTL epitopes with higher binding affinity $(\leq 1.5)$ with MHC-II were selected for the final

3 multi-epitope vaccine construct

4 


\section{Table 4 (on next page)}

B cell specific epitopes and their score as predicted and obtained from the immune epitope database 


\begin{tabular}{cccc} 
S/N & Position & Epitope & Score \\
\hline $\mathbf{1}$ & 170 & RVKAGPGPGPRKVSQIKIVT & 1 \\
$\mathbf{3}$ & 247 & SVCHPIIGPGPGDIVSQQQA & 1 \\
$\mathbf{4}$ & 370 & LDSIGPGPGGCAFQAAICSP & 1 \\
$\mathbf{5}$ & 328 & PAFKVKGPGPGDQPRKVSQI & 1 \\
$\mathbf{6}$ & 285 & AMESVCHPIGPGPGNRSLVR & 1 \\
$\mathbf{7}$ & 411 & LDSGPGPGRGCAFQAAICSP & 1 \\
$\mathbf{8}$ & 191 & RVKGPGPGQPRKVSQIKIVT & 1 \\
$\mathbf{9}$ & 145 & TSSTDGDLVGPGPGRKVSQI & 1 \\
$\mathbf{1 0}$ & 307 & KVLDSIAGPGPGCAFQAAIC & 1 \\
$\mathbf{1 1}$ & 226 & KVLDSIAAGPGPGVSQQQAM & 1 \\
$\mathbf{1 2}$ & 442 & LTTKELVKAFTRGPGPGTRL & 1 \\
$\mathbf{1 3}$ & 349 & IVTSRGPGPGFNRSLVRIRK & 1 \\
$\mathbf{1 4}$ & 467 & NVPAYDLGPGPGTDIVSQQQ & 1 \\
\hline $\mathbf{1 5}$ & $\mathbf{5 4}$ & IEEYAAYNSKNAVEEYAAYF & 0.994 \\
\hline $\mathbf{2 0 m}$ & 14 & 0.753 \\
\hline
\end{tabular}

*only 20 mers epitopes with equal and above 0.99 score were selected 